\title{
Carbon Nanotube Network Structure Induced Shape Memory Behavior Changes of Liquid Crystalline Polyurethane
}

\author{
Yueting $\mathrm{Li}^{1,2}$, Huiqin $\mathrm{Lian}^{1, *}$, Wei Chang ${ }^{1,2}$, Benzhe $\mathrm{Wu}^{1}$, Yupeng $\mathrm{Liu}^{1}$, Junlin $\mathrm{Ma}^{1}$, Junwei Ma ${ }^{1}$, Jierui Wang ${ }^{1}$ \\ ${ }^{1}$ Beijing Key Lab of Special Elastomer Composite Materials, College of Materials Science and Engineering, Beijing Institute of \\ Petrochemical Technology, Beijing 102617, China \\ ${ }^{2}$ College of Materials Science and Engineering, Beijing University of Chemical Technology, Beijing 100029, China
}

\begin{abstract}
Liquid crystalline polyurethane (LCPU) was used to develop shape memory nanocomposites. The carbon nanotubes were introduced by melt mixing to improve the shape memory property of the LCPU matrix. The dispersion of CNTs and shape memory behaviours of the nanocomposites were also studied. The XRD shows that the CNTs have a good dispersion in the LCPU matrix. The nanocomposites shows a better recovery ratio with the CNTs increase.
\end{abstract}

\section{Introduction}

The liquid-crystal polymer (LCP) is a thermoplastic polymer material with unique structural and physical properties[1]. They have some advantages over general polymers. The synthesis of liquid crystalline polyurethane (LCPU) was first reported by Limure et al.[2]. Since then, these LCPs have drawn considerable attention because of their excellent commercial and scientific applications[3]. Muti-walled carbon nanotube (MWCNTs) have attracted wide attention of their superior properties such as mechanical strength, large surface areas[4], and aspect ratios as well as their high thermal conductivities[5]. In this work, the liquid crystalline polyurethanes which contained large group of carboxyl in the reaction of 4,4'-methylenediphenyl diisocyanate (MDI), 1,6-hexanediol (HDO), 2,2dimethylol propionic acid (DMPA) and polytetramethylene ether glycol (PTMG) and then LCPU/MWCNTs nanocomposite were prepared. We show that carbon nanotube can contribute to the shape memory property of the LCPU. We observed increase in the recovery rate and recovery temperature of LCPU composites prepared with low amounts of CNTs.

\section{Experimental and methods}

\subsection{Materials and preparation of composites}

The matrix of the studied nanocomposites was a liquid crystalline polyurethane. The hard segment is formed by diisocyanate and chain extending short chain diol, and the soft segment is formed by the long chain diol. The aromatic diisocyanate is diphenylmethane diisocyanate (MDI), the chain extender is a short-chain diol, 1,6hexanediol (HDO) and 2,2-dimethylol propionic acid (DMPA) and long chain diol is polytetramethylene ether glycol (PTMG). The composition was $48 \%$ hard segment and $52 \%$ soft segment. The structure is shown in Fig.1.

Multiwall Carbon Nanotubes (MWCNT) were purchased from CNano Technology Co., Ltd (Beijing, China). The purity of MWCNT was more than $95 \%$, and average specified diameters of $\mathrm{D}=7-11 \mathrm{~nm}$, Lengths of $\mathrm{L}=10-20 \mu \mathrm{m}$. The nanocomposites were prepared dispersing the nanoparticles by melt mixing method. In this work, the content of MWCNT was varied from $0.1 \mathrm{wt} \%, 0.3 \mathrm{wt} \%$ and $0.5 \mathrm{wt} \%$. The sample notation was defined as LCPUxC, where $\mathrm{x}$ represented the content of MWCNT in the composites.

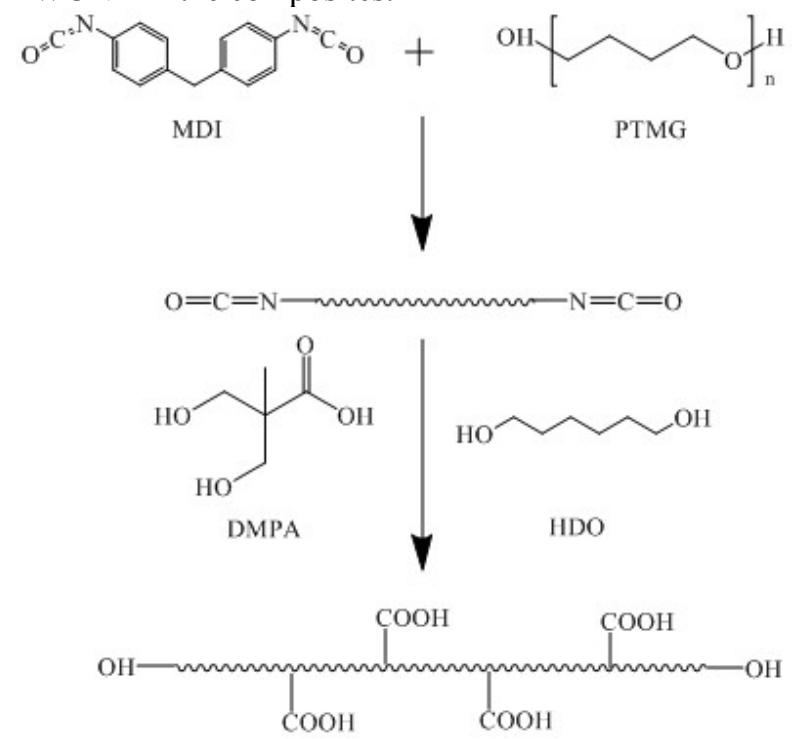

Figure 1. The structure of the liquid crystalline polyurethane

\subsection{Measurements}

The structure of the films was analyzed by a Thermo Scientific Nicolet 6700-OMNI Smart Accessory Spectrum Fourier transform infrared (FTIR) spectrometer. Spectra were collected in the mid-infrared range from 500 to $400 \mathrm{~cm}^{-1}$ in the ART mode. X-ray Diffraction (XRD) spectra of the films were collected on Bruker D8 Focus. Data were collected between $10.9378^{\circ}$ and $80.0067^{\circ}$ with a step of $0.06^{\circ}$ and a dwell time of $1.5 \mathrm{~s}$. To study the shape memory property of the samples, four specimens of LCPU and composites were cut from the films, and rectangular samples of $10 \mathrm{~mm}$ in width, $50 \mathrm{~mm}$ in length were cut for shape memory characterization. Four rectangular samples were heated in dryer for $5 \mathrm{~min}$ in order to allow relaxation of the LCPU chains, then removed them from the dryer into the cold water and folded in half for 10min to maintain the deformation. All the samples were put back to the dryer at $90^{\circ} \mathrm{C}$, and 
recorded the shape recovery by camera. In addition, the shape recovery of LCPU0.5C was tested at different temperature $\left(60^{\circ} \mathrm{C}, 70^{\circ} \mathrm{C}\right.$ and $\left.80^{\circ} \mathrm{C}\right)$ for comparison. In order to have a quantitatively evaluation of the shape memory property of the samples, the shape recovery ratio (Rr) was calculated according to Eq.(1), where Am was the angle after reheated, and Ap is $180^{\circ}$ which was the original anger (the angle was read from the protractor below the samples).

Shape recovery rate $(\%)=A_{m} / 180^{\circ} \times 100$

\section{Results and discussion}

\subsection{Structures of the pristine MWCNT, the LCPU and the nanocomposites}

The infrared spectra in Fig.2 show some typical functional groups of LCPU. The most relevant bands corresponded to N-H stretching at $3174-3160 \mathrm{~cm}^{-1}$ which indicate that polyurethanes are capable of forming hydrogen bonds in which the N-H group of the urethane linkage is the proton donor[6]. The bands near $1410 \mathrm{~cm}^{-1}$ are assigned to the $\mathrm{CH}_{2}$ groups. The absorbance in the region near $1735 \mathrm{~cm}^{-1}$ is assigned to the stretching vibration of free $\mathrm{C}=\mathrm{O}$ groups in urethane. And the absence of NCO groups at $2250-2279 \mathrm{~cm}^{-1}$ indicates that the reaction proceeded until complete conversion of the isocyanate[7]. In addition, IR spectra showed the characteristic $\mathrm{C}-\mathrm{O}$ and N-CO-O stretching (1219$1218 \mathrm{~cm}^{-1} ; 1058-1056 \mathrm{~cm}^{-1}$ ) bands in polyurethanes. These spectral features showed that LCPU was successfully synthesized. Then compared with the Fig.2(b), it indicates that the composites were synthesized.

XRD spectra of pristine polyurethane and LCPU/MWCNTs nanocomposite films are shown in Fig. 3. LCPU and composite film shows that semicrystalline and characterized by two peaks. The position of pristine LCPU and MWCNTs peak is very close to each other. The position of MWCNTs peak observed at $2 \theta=25$ as has been reported in literature[8]. LCPU/MWCNTs shows one peak around $20.42^{\circ}$ and the pristine LCPU show diffraction peak at $2 \theta=20.53^{\circ}$ due to the presence of soft segments crystals. The X-ray pattern of the films shows mixing of the peaks appearing for the MWCNTs and the LCPU. LCPU containing MWCNTs shows much lower diffraction intensity as compared to pristine LCPU.

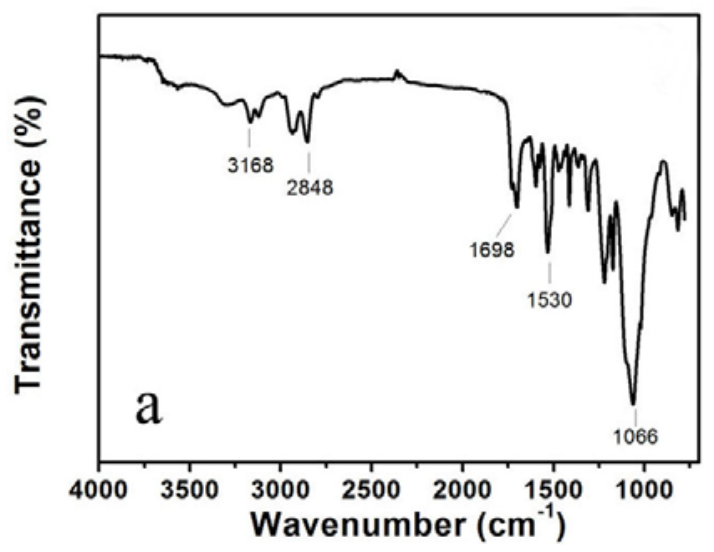

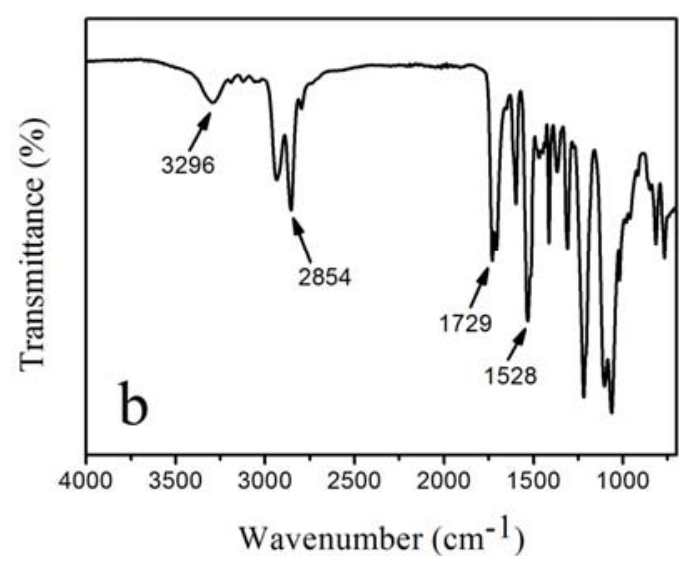

Figure 2. FTIR spectra of (a) MWCNT and (b) LCPU

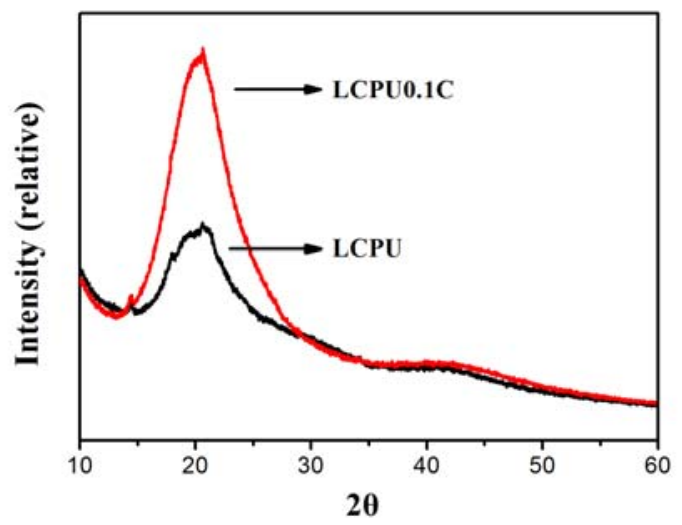

Figure 3. XRD spectra of (a) MWCNT and (b) LCPU

\subsection{Thermal properties}

The polymer matrix used in this work is a kind of liquid crystalline polyurethane elastomer consisting of hydrogen bonded urethane linkages and the easter (-COO-) groups of polyol soft segments. The fixed and reversible phase play crucial roles in the shape memory property of SMPs. In this work, the fixed phases are hydrogen bonded urethane linkages. Then the amorphous chain act as the reversible phase[9]. In the study of the shape memory property, the films were deformed above the glass transition temperature $\left(\mathrm{T}_{\mathrm{g}}\right)$ of the reversible phase, and the temporary shape was then frozen in the ice atmosphere. When the temperature is above the $T_{g}$, the shape of the films recovers. So the glass transition of the amorphous phase act as a switch for the control of the shape fixity and recoverability.

From the DSC curves in the Fig.4, all the samples show the $\mathrm{T}_{\mathrm{g}}$ around $50^{\circ} \mathrm{C}$, indicating the nanotube have no effect on the $T_{g}$ of the polymer chians[10]. Generally, the incorporation of nanoparticles constrains the movement of the molecular chains, causing the increasing of $\mathrm{T}_{\mathrm{g}}$ as reported[11]. 


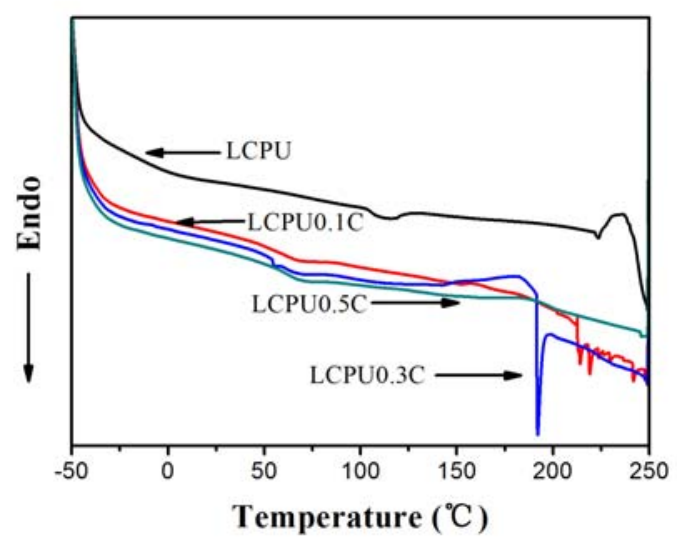

Figure 4. DSC traces of LCPU and its nanocomposites

\subsection{Shape memory properties}

The shape memory properties can be characterized by shape fixity $\left(R_{f}\right)$ and shape recovery ratio $\left(R_{r}\right)[11]$. The $\mathrm{R}_{\mathrm{f}}$ of an SMP material is influenced by a major factors[12]. It is the amount of 'unlocked' chains and the chain segments. If an deformation is happened, all chains are in the same level of deformation in the stretching process[13]. However, a fraction of the chains and chain segments still preserve mobility even the temperature is lower than the $\mathrm{T}_{\mathrm{g}}$. These 'unlocked' chains produced an instantaneous retractive force upon removal of the tensile load with the elasticity[14]. But this retractive force cannot cause the instantaneous recovery of the shape as the fraction of mobile chains is usually low.

The influence from the content of MWCNT on shape memory property was shown in Table 1 . It was easy to discover that with the increase of MWCNT, the shape recovery rate increased and time decreased. That was to say that the MWCNT can contribute to the shape memory property. It was because that the MWCNT can transfer the heat effectively which was the main factor affecting the shape memory property.

In order to observe the influence of temperature on the shape memory property, a comparison between the different temperature and recovery time was reported in Fig.5.

The LCPU0.5C sample recovered totally at these four temperature, and the recovery time decreased with the temperature increased. With the increase of temperature, the movement of polymer chains was accelerated, so the shape at $90^{\circ} \mathrm{C}$ recovered faster than other samples.

Images of the shape recovery tests of the LCPU and LCPU0.5C nanocpmposite are presented in Fig.6 and Fig.7. Obviously, the LCPU0.5C have faster recovery which means that the nanocomposites has higher elastic ratio and more energy stored in the temporary shape.
Table 1. The shape recovery rate and time of samples at $90^{\circ} \mathrm{C}$

\begin{tabular}{|c|c|c|}
\hline Sample & Recovery rate (\%) & Time (s) \\
\hline LCPU & 61.56 & 79.19 \\
\hline LCPU0.1C & 93.28 & 63.67 \\
\hline LCPU0.3C & 97.92 & 20.52 \\
\hline LCPU0.5C & 100.00 & 10.87 \\
\hline
\end{tabular}

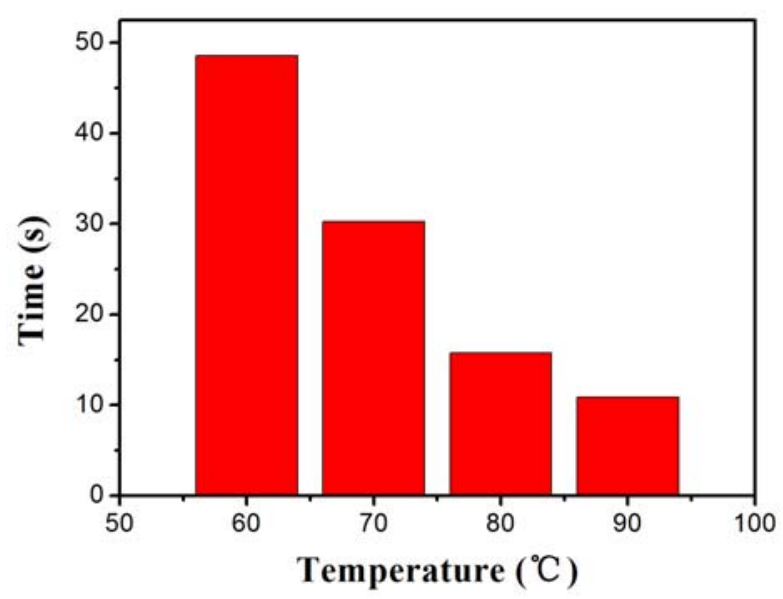

Figure 5. The shape recovery time of LCPU0.5C in different temperature

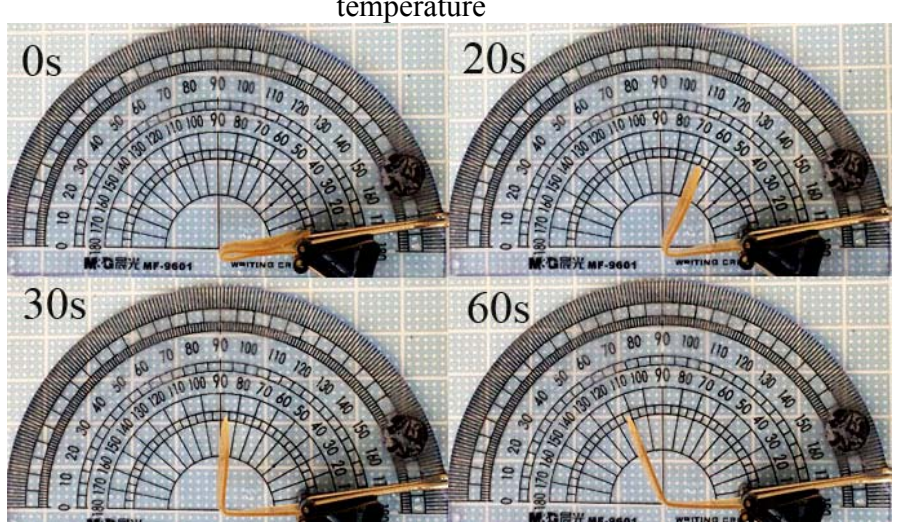

Figure6. The shape recovery images of LCPU 


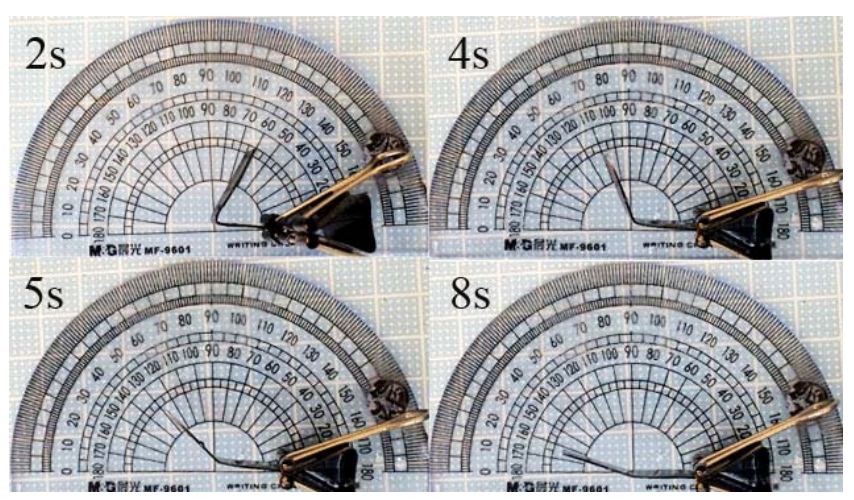

Figure 7. The shape recovery images of LCPU0.5C

\section{Summary}

In this paper, a series of LCPU/MWCNTs composites has been synthesized. H-bonding and crystallinity, which affected the thermal properties, were showed in FTIR and XRD respectively. The XRD spectra shows that the MWCNTs has been dissolved well in the LCPU. The LCPU/MWCNTs showed good performance while the molar ratio of MWCNTS was elevated. As the ratio increased, the shape memory property was increased. The shape recovery rate of LCPU0.5C can reach $100 \%$ and in only $10.87 \mathrm{~s}$.

\section{Acknowledgements}

This work is supported by the Graduate student innovation project (NO.15033981002/040), the Natural Science Foundation of China (NSFC, Nos. 51063009, 21271031, 51203012), the Beijing Natural Science Foundation of China (Nos. 2132009, 2122015), undergraduate Research Training Program of Beijing (2015J00002) and the project about the promotion of innovation in Beijing universities funded by Beijing Municipal commission of education of China.

\section{References}

[1] Bagheri, M. and Z. Pourmoazzen, Synthesis and properties of new liquid crystalline polyurethanes containing mesogenic side chain. Reactive and Functional Polymers, 2008. 68(2): p. 507-518.

[2] Lal, S. and J.K. Quamara, Effects of $100 \mathrm{MeV} \mathrm{O7+}$ ion beam irradiation on the optical, chemical and structural properties of NCO-terminated polybutadiene based liquid crystalline polyurethane. Vacuum, 2014. 105: p. 7-10.

[3] Fang, L., et al., Synthesis of well-defined easily crosslinkable azobenzene side-chain liquid crystalline polymers via reversible additionfragmentation chain transfer polymerization and photomechanical properties of their postcrosslinked fibers. European Polymer Journal, 2015. 69: p. 592-604.

[4] Gu, S., et al., Carbon nanotube-polyurethane shape memory nanocomposites with low trigger temperature. European Polymer Journal, 2013. 49(12): p. 3867-3877.

[5] Chen, J., et al., Carbon nanotube network structure induced strain sensitivity and shape memory behavior changes of thermoplastic polyurethane. Materials \& Design, 2015. 69: p. 105-113.

[6] Huang, K., S. Pisharath, and S.-C. Ng, Preparation of polyurethane-carbon nanotube composites using 'click' chemistry. Tetrahedron Letters, 2015. 56(4): p. 577-580.

[7] Nam, D.H., et al., Synergistic strengthening by load transfer mechanism and grain refinement of CNT/Al-Cu composites. Carbon, 2012. 50(7): p. 2417-2423.

[8] Singh, R., M.S. Gaur, and R.K. Tiwari, Development of polyurethane multiwall carbon nanotubes (MWCNTs) novel polymeric nanodielectric material. Journal of Electrostatics, 2015. 76: p. 95-101.

[9] Rosu, D., N. Tudorachi, and L. Rosu, Investigations on the thermal stability of a MDI based polyurethane elastomer. Journal of Analytical and Applied Pyrolysis, 2010. 89(2): p. 152-158.

[10] Silvestre, N., B. Faria, and J.N. Canongia Lopes, Compressive behavior of CNT-reinforced aluminum composites using molecular dynamics. Composites Science and Technology, 2014. 90: p. 16-24.

[11] Fonseca, M.A., et al., Shape memory polyurethanes reinforced with carbon nanotubes. Composite Structures, 2013. 99: p. 105-111.

[12] Moon, S., F. Cui, and I.J. Rao, Constitutive modeling of the mechanics associated with triple shape memory polymers. International Journal of Engineering Science, 2015. 96: p. 86-110.

[13] Sujithra, R., S.M. Srinivasan, and A. Arockiarajan, Shape recovery studies for coupled deformations in an epoxy based amorphous shape memory polymers. Polymer Testing, 2015. 48: p. 1-6.

[14] Raasch, J., et al., Characterization of Polyurethane Shape Memory Polymer Processed by Material Extrusion Additive Manufacturing. Additive Manufacturing. 